Check for updates

Cite this: RSC Adv., 2017, 7, 37426

\title{
Drug loaded poly(glycerol sebacate) as a local drug delivery system for the treatment of periodontal disease
}

\begin{abstract}
Bo Yang, Wei Lv and Ying Deng (iD)*
Periodontal disease is a widely distributed disease worldwide. It affects life quality and causes many health problems. The cause of periodontal disease is bacterial infection. The key to treating periodontal disease is killing bacteria and thus using a local antibiotic agent is a good way of treating periodontal disease. In our work, we loaded berberine and chlorhexidine into biodegradable elastomer poly(glycerol-sebacate) (PGS) using a swell drug loading method. Drug(s) showed sustained release properties in vitro. Mechanical testing showed that neither drug loading method nor loaded drug(s) changed the Young's modulus and maximum strain of PGS. Loaded drugs changed the surface wettability and cell compatibility. The general antimicrobial test showed that chlorhexidine loaded PGS groups were good against typical Gram positive bacteria, Gram negative bacteria and antibiotic-resistant bacteria. Berberine loaded PGS groups showed good antibacterial ability against periodontal disease pathogens. These results indicate that our drug loaded PGS can be used in the treatment of periodontal disease.
\end{abstract}

Received 7th March 2017

Accepted 14th June 2017

DOI: 10.1039/c7ra02796f

rsc.li/rsc-advances

Many antibiotics including tetracycline, oxytetracycline,

\section{Introduction}

Periodontal disease is a widely distributed oral disease which affects around $10 \%$ to $15 \%$ of the adult population worldwide. ${ }^{1}$ It can cause many health problems including bad breath, gum bleeding and teeth loss. ${ }^{2-4}$ Research also indicates that periodontal disease is able to increase the chance of getting coronary artery disease or other systemic problems. ${ }^{5}$ Several factors including dining habits, environment, and smoking contribute to the generation of periodontal disease. ${ }^{5}$ The main cause of periodontal disease is bacterial infection and biofilm formation. Over 300 species of bacteria have been involved in this process. ${ }^{6}$ Among these bacteria species, Porphyromonas gingivalis and Aggregatibacter actinomycetemcomitans are considered as the most important pathogens and thus have been used in many periodontal disease-related research studies. ${ }^{7-10}$ Different stages of periodontal disease have different treatments. A healthy diet and toothbrush are able to prevent periodontal disease effectively; deep cleaning is used to clean the calcified biofilm (tartar) and thus is able to prevent the development of periodontal disease. Surgical treatments including flap surgery and bone/tissue grafts are the final way to treat late stages of periodontal disease., ${ }^{3,11,12}$ Using antibiotics and antibiotic implants is an important method to treat periodontal disease. Thus it is widely used in treating early stages of periodontal disease and post-surgical recovery. ${ }^{13}$

Biomedical Engineering Program, University of South Dakota, 4800 North Career Avenue, Sioux Falls, South Dakota 57107, USA. E-mail: Ying.Deng@usd.edu; Fax: +1 605-367-7836; Tel: +1 605-275-7469 chloramphenicol, ciprofloxacin and chlorhexidine were used in many products for the treatment of periodontal disease. ${ }^{14-16}$ Chlorhexidine has a wide antimicrobial spectrum against Gram positive, Gram negative bacteria and fungi. ${ }^{17}$ Because of its wide antimicrobial spectrum and high antimicrobial efficiency, it is highly effective against a broad spectrum of Gram positive, Gram negative bacteria and fungi. Chlorhexidine is widely used in the treatment of periodontal disease and is considered as "gold standard" in the field. ${ }^{16,17}$

Berberine is a quaternary alkaloid extracted from many herbs like Hydrastis canadensis (goldenseal), Coptis chinensis (goldenthread) and Berberis aquifolium (Oregon grape). ${ }^{18}$ It has been used in traditional Chinese and Ayurvedic medicine for centuries to treat diseases such as diarrhea, eye inflammation, and gastric disorders. ${ }^{18}$ Modern researches reveal that berberine has many pharmacological effects including antimalarial, anti-secretory, anti-inflammatory and anti-cancer activity. ${ }^{19-21}$ Berberine has a wide range of antimicrobial spectrum against Gram positive bacteria, Gram negative bacteria, fungi and antibiotic-resistant bacteria like methicillin-resistant S. aureus (MRSA) ${ }^{19,22,23}$ Although the minimum inhibitory concentration (MIC) of berberine is higher than many commonly used antibiotics, ${ }^{24}$ it can be used to enhance antibiotic's antimicrobial ability. ${ }^{25}$ Berberine is a relatively safe drug. ${ }^{26}$ The antimicrobial, anti-inflammatory, and hemostatic potential made berberine useful in wound dressing applications. ${ }^{27}$ One recent study also indicated that berberine might have the ability to slow the periodontal tissue degradation process. ${ }^{28}$ This indicated that berberine could be used as 
a good drug additive with chlorhexidine in the treatment of periodontal disease.

Poly(glycerol sebacate) (PGS) was firstly invented in 2002 by Wang Y. et al. ${ }^{29}$ It has a 3-D cross-linked structure composited by equimolar of glycerol and sebacate acid..$^{29,30}$ PGS undergoes an enzymatic surface degradation with more than $70 \%$ mass degraded for 35 days in vivo. ${ }^{31-33}$ For the in vitro studies, PGS undergoes approximately $20 \%$ of the mass loss after 14 days in PBS solution. ${ }^{34}$ The difference is most likely due to the fact that enzymes and macrophages attack the PGS polymer and mediate the degradation in vivo. However, the degradation rate also depends on the curing temperature/time, ratio of sebacic acid and glycerol as well as degree of acrylation. ${ }^{35}$ One unique property of PGS is its elastic mechanical property, which can also be controlled during the synthesis process. ${ }^{30,36}$ Because of its unique mechanical property, good biocompatibility and biodegradation property, PGS was used in many tissue engineering and drug delivery studies..$^{30-33,36-39}$

In our work, we developed a swell drug loading method and loaded berberine and chlorhexidine into PGS. The drug loaded PGS showed good biological function while kept the same mechanical property with non-drug loaded PGS. The result showed the feasibility of our swelling drug loading method. Also, drug loaded PGS, especially berberine and chlorhexidine loaded PGS (BC-PGS) can be a good candidate for the treatment of periodontal disease.

\section{Material and methods}

\subsection{Materials}

Bacteria strains Staphylococcus aureus (ATCC 6538), Escherichia coli (ATCC 15597), methicillin-resistant Staphylococcus aureus (MRSA, ATCC BAA-811), Porphyromonas gingivalis (ATCC 33277) and Aggregatibacter actinomycetemcomitans (ATCC 29522) were purchased from American type culture collection (ATCC, Manassas, VA). Human gingival fibroblast cell (HGF-1, ATCC CRL2014) was purchased from American type culture collection (ATCC, Manassas, VA). All reagents used in our work including glycerol, sebacic acid, berberine chloride, chlorhexidine and ethanol were from Sigma-Aldrich (Sigma-Aldrich, St Louis, MO) unless otherwise specified.

\subsection{PGS synthesis}

Poly(glycerol sebacate) (PGS) was synthesized following the reported two-step synthesis method. ${ }^{29}$ Briefly, equimolar of glycerol and sebacic acid were mixed evenly and put into a vacuum oven (Fisher Isotemp Vacuum Oven Model 281A, Fisher Scientific, Waltham, MA) at $120^{\circ} \mathrm{C}$ in $\mathrm{N}_{2}$ atmosphere for 24 hours for the synthesis of PGS pre-polymer (pre-PGS). Pre-PGS was then cross-linked at $120{ }^{\circ} \mathrm{C}$ under vacuum for 72 hours to make the 3D cross-linked PGS elastic polymer. After synthesis, PGS was cut into $10 \mathrm{~mm} \times 10 \mathrm{~mm} \times 4 \mathrm{~mm}$ cubes and balanced in distilled (DI) water for 3 days before use.

\subsection{PGS swelling test}

PGS was synthesized, buffered and cut into $10 \mathrm{~mm} \times 10 \mathrm{~mm} \times$ $4 \mathrm{~mm}$ cubes as mentioned above. PGS cubes were weighted $\left(W_{0}\right)$ before swelling test. Ethanol and acetone were selected for swelling test because they are commonly used, less toxic class 3 solvents in pharmaceutical industry. ${ }^{40,41}$ PGS cubes were immersed in each organic solvent for 48 hours until they swelled to equilibrium, then the excessive surface solvent was removed with filter paper, the fully swollen samples were weighted again $\left(W_{1}\right)$. The swelling behavior $\left(\mathrm{SB}_{\mathrm{w}}(\%)\right)$ was calculated based on weight change with the following equation:

$$
\mathrm{SB}_{\mathrm{w}}(\%)=\frac{W_{1}-W_{0}}{W_{0}} \times 100 \%
$$

PGS swelling behavior in DI water and phosphate buffered saline (PBS) were also tested for reference. The swelling tests were repeated three times until there was no further weight increase. $^{42}$

\subsection{Drug loading}

Like most elastomer, PGS swells in many organic solvents. ${ }^{43,44}$ This property was used for drug loading in our work. Drugs can be loaded into PGS using various organic solvents. In our current work, we chose berberine and chlorhexidine as model drugs and ethanol as a drug-loading solvent because of its low toxicity. ${ }^{40,41} 10 \mathrm{mg} \mathrm{ml}^{-1}$ of berberine chloride and $10 \mathrm{mg} \mathrm{ml}^{-1}$ of chlorhexidine were prepared in $10 \mathrm{ml} 200$ proof ethanol for a saturated solution. PGS cubes were then immersed into saturated drug/ethanol solution for drug loading at $37^{\circ} \mathrm{C}$ for 48 hours. Drug-loaded PGS cubes were then washed with DI water to remove surface attached $\operatorname{drug}(\mathrm{s})$ and residual solvent, vacuum dried at room temperature for later use. In our work, berberine loaded PGS (B-PGS) cubes, chlorhexidine loaded PGS (C-PGS) cubes, and berberine-chlorhexidine loaded PGS (BCPGS) cubes were prepared with saturated drug ethanol solution respectively and PGS cubes treated with same drug loading method without drug were employed as a control.

\subsection{FTIR characterization}

The PGS polymer, drugs and the drug loaded samples were characterized using Fourier transform infrared spectroscopy (FTIR). A Nicolet 6700 FTIR spectrometer (Thermo Scientific) equipped with an attenuated total reflection (ATR) accessory was used. Unless otherwise mentioned, all IR spectra were collected using 512 scans at $4 \mathrm{~cm}^{-1}$ spectral resolution.

\subsection{Drug content and release test}

Drug content of B-PGS, C-PGS and BC-PGS was measured by breaking down and dissolving samples cubes with $10 \mathrm{ml}$ of 2,2,2-trifluoroethanol (TFE). Berberine and chlorhexidine concentration was determined by measuring the UV absorption of the supernatant at $344 \mathrm{~nm}$ (ref. 45 ) and $254 \mathrm{~nm},{ }^{46}$ respectively using a micro-plate reader (Tecan infinite M200, Tecan Group Ltd. Männedorf, Switzerland). 
Sterile PBS was used for drug release test. One cube of treated PGS sample was put into a glass vial containing $10 \mathrm{ml}$ sterile PBS. The PGS sample containing glass vial was then put into a mechanical shaker to perform the drug releasing study at $37^{\circ} \mathrm{C}, 150 \mathrm{rpm}$. Sterile PBS was changed daily, and released drug was measured using the same method as in drug content test.

\subsection{Mechanical property test}

Synthesized PGS were cut into $50 \mathrm{~mm} \times 10 \mathrm{~mm} \times 4 \mathrm{~mm}$ strips. Drug(s) were loaded into PGS strips using the same method while an untreated group was prepared without any drug loading treatments. For the mechanical test, five groups of samples (PGS, B-PGS, C-PGS, BC-PGS and untreated) were mounted on the sample loader of MTS insight electromechanical testing system (MTS systems corporation, Eden Praire, MN). The strain rate was $50 \mathrm{~mm} \mathrm{~min}^{-1}$, and all samples were elongated to failure. Young's modulus and strain at break were measured by running MTS tensile method with five groups of samples. Modulus of elasticity was extracted by the equation:

$$
E=\frac{\text { stress }}{\text { strain }}
$$

In addition, the cross-linking density $(n)$ was calculated according to the theory of rubber elasticity using the following equation:

$$
n=\frac{E_{0}}{3 R T}
$$

where $n$ represents the number of active network chain segments per unit volume $\left(\mathrm{mol} \mathrm{m} \mathrm{m}^{-3}\right), E_{0}$ represents Young's modulus, $R$ is the universal gas constant, $T$ is the absolute temperature $(\mathrm{K}) .^{47}$

\subsection{Surface wettability test}

Surface wettability was determined by contact angle goniometry using VCA optima contact angle analysis system (AST products, Inc., Billerica, MA). In this test, one drop ( $2 \mu \mathrm{l})$ of DI water was dropped onto the horizontal surface of a sample cube. Photos were taken using the contact angle analysis system after 15 seconds of contact and contact angles were measured with associated VCA optima software.

\subsection{Antimicrobial test}

The general antimicrobial test was taken following ASTM standard E2149-10. ${ }^{48}$ E. coli was cultured in LB broth (Lennox, Fisher Scientific, Pittsburgh, PA) overnight. S. aureus and MRSA was cultured in tryptic soy broth (Fluka analytical, SigmaAldrich, St Louis, MO) overnight. All bacteria were grown to OD470 $=0.30-0.35$ and was further diluted 100 times with PBS. PGS, B-PGS, C-PGS and BC-PGS cubes were put into $20 \mathrm{ml}$ diluted bacteria solution and shook vigorously at Isotemp shaker (MAXQ 4450, Thermo Scientific, Waltham, MA) at $37{ }^{\circ} \mathrm{C}$. The colony formation unit (CFU) of supernatant was tested after 1 and 4 hours to determine the antimicrobial activity of four groups of samples.
The anti-periodontal bacteria test was taken using KirbyBauer test (inhibition zone). Periodontal disease pathogens $P$. gingivalis and A. actinomycetemcomitans were cultured in brain heart infusion (BHI) broth (Becton, Dickinson and Company, Sparks, MD) for two days in an anaerobic environment $\left(5 \% \mathrm{CO}_{2}\right.$, $5 \% \mathrm{H}_{2}$ and $90 \% \mathrm{~N}_{2}$ ). Bacteria were then diluted 10 times with PBS and spread evenly on a BHI agar plate. Four groups of samples were punched to $6 \mathrm{~mm}$ tablets by biopsy punched and put onto bacteria agar plates. Agar plates were then cultured in an anaerobic environment for 2 days until a clear inhibition zone were observed.

\subsection{Cell compatibility test}

Human gingival fibroblasts cell (HGF-1, ATCC@ CRL-2014 ${ }^{\mathrm{TM}}$ ) was used for the cell compatibility because it is the most abundant cell in human gingival tissue. ${ }^{49,50}$ HGF-1 cells were cultured in DMEM/high glucose supplemented with 1\% non-essential amino acids (Fisher Scientific, Pittsburgh, PA), 1\% antibiotics (Fisher Scientific, Pittsburgh, PA), and 10\% fetal bovine serum (Sigma-Aldrich, St Louis, MO). Four groups of samples were sterilized by exposing to ultraviolet radiation (UV) for $30 \mathrm{~min}$ and were balanced in cell culture media for 7 days in a 24 well plate before the test. HGF-1 cells were then seeded onto the surface of the membranes at a concentration of 100000 cells per well. PGS cubes were taken out and fixed with $2.5 \%$ glutaraldehyde at $4{ }^{\circ} \mathrm{C}$ overnight. Samples were then permeabilized with $0.5 \%$ triton $\mathrm{X}$ 100 for 10 minutes at room temperature. Permeabilized cells were stained with $100 \mathrm{nM}$ rhodamine phalloidin (Molecular Probes Life Technologies, Eugene, OR) in the dark at room temperature for 30 minutes. After rhodamine phalloidin stain, samples were treated with $0.3 \%(\mathrm{w} / \mathrm{w})$ Sudan Black for 30 minutes to reduce background emission. ${ }^{51}$ Fluorescence images were taken by Axiovert 200 fluorescence microscope (Axiovert 200, Carl Zeiss AG, Jena, Germany).

\subsection{Statistical analysis}

All quantitative tests were carried out with three parallel samples, with results presented in the form of mean \pm standard deviation (S.D.) unless otherwise specified. The statistical significance of the data obtained was analyzed by one-way ANOVA. Probability values of $p<0.05$ were interpreted as statistically significant.

\section{Results}

\subsection{PGS swelling and drug loading}

PGS showed different swelling behavior in DI water, PBS, ethanol, and acetone as shown in Table 1. Compare to DI water and PBS, PGS swelled more in organic solvents. The integrity of PGS cubes is different after swelling treatment with different solvents. While PGS cube surface remains integrated and smooth after treated with DI water, PBS, and ethanol, the surface showed many cracks after the acetone treatment (data not shown). In this way, acetone will not be used for drug loading because it cannot keep the cube integrity, which may, in turn, affect the mechanical property. 
Table 1 Swelling property of PGS in different solvents

\begin{tabular}{|c|c|c|c|c|}
\hline Solvent & Water & PBS & Ethanol & Acetone \\
\hline Swelling & $3.82 \% \pm 0.25 \%$ & $3.41 \% \pm 0.19 \%$ & $55.22 \% \pm 0.51 \%$ & $113.98 \% \pm 1.95 \%$ \\
\hline
\end{tabular}

Table 2 Expected and actual drug loading efficiency of B-PGS and CPGS

\begin{tabular}{|c|c|c|c|}
\hline & $\begin{array}{l}\text { Expected drug } \\
\text { loading }\end{array}$ & $\begin{array}{l}\text { Tested drug } \\
\text { loading }\end{array}$ & $\begin{array}{l}\text { Drug loading } \\
\text { efficiency }\end{array}$ \\
\hline Berberine in B-PGS & $0.402 \%$ & $0.699 \%$ & $173.71 \%$ \\
\hline Chlorhexidine in C-PGS & $0.019 \%$ & $0.042 \%$ & $221.49 \%$ \\
\hline
\end{tabular}

Using tested PGS swelling behavior $\left(\mathrm{SB}_{\mathrm{w}}\right)$, drug loading concentration $\left(c_{1}\right)$ and solvent density $(\rho)$, we can calculate expected drug loading (EDL $(\mathrm{w} / \mathrm{w})$ ) with the following concentration:

$$
\operatorname{EDL}(\mathrm{w} / \mathrm{w})=\frac{\mathrm{SB}_{\mathrm{w}}}{\rho} \times c_{1} \times 100 \%
$$

The expected drug loading is calculated by assuming that drugs and material have no interaction, while the only mechanism for drug loading is the swelling process. However, as shown in Table 2, we noticed that tested drug loading is higher than expected drug loading for both berberine and chlorhexidine. This indicates that PGS may have interactions with berberine and chlorhexidine during the loading process. This result was further confirmed by FTIR characterization.

\subsection{FTIR}

Fig. 1 shows the FTIR spectra of berberine, chlorhexidine, BCPGS, B-PGS, C-PGS, PGS respectively. The peaks at 2927-2850 $\mathrm{cm}^{-1}$ were attributed to alkene $\left(-\mathrm{CH}_{2}\right)$ groups. The peaks at 1160 and $1731 \mathrm{~cm}^{-1}$ were assigned to the $\mathrm{C}-\mathrm{O}$ and $\mathrm{C}=\mathrm{O}$, respectively, while $\mathrm{CH}_{3}$ bending appeared at $1378-1456 \mathrm{~cm}^{-1}$. The O-H stretch peak was shown at $940 \mathrm{~cm}^{-1}$ while the peak at $1416 \mathrm{~cm}^{-1}$ was assigned to the $\mathrm{O}-\mathrm{H}$ bending. The two peaks observed on PGS spectra at 1291 and $1218 \mathrm{~cm}^{-1}$ were assigned to the stretching of $\mathrm{C}-\mathrm{O}$ groups. After drug had loaded on PGS polymer, several changes were observed in the FTIR spectrum. The spectra of B-PGS, C-PGS and BC-PGS showed the characteristic peaks of PGS with no change in the peak positions observed when compared to that of the control PGS. However, the peaks for the berberine and chlorhexidine were not evident in these spectra. This suggested that the berberine and chlorhexidine were not present on the surface of PGS cubes.

It is shown that the peaks at 1291, 1218 and $940 \mathrm{~cm}^{-1}$ significantly reduced their intensity after drug loading. This is an indication of the interactions of the drugs and PGS polymer.

\subsection{Drug release behavior}

We did drug release test in sterile PBS to minimize the PGS degradation. $^{32,33}$ The release curve of berberine in B-PGS and BC-PGS was shown in Fig. 2. Typical two-phasic release patterns were observed: (i) at early time points (in 10 days), the release rate was high (initial "burst effect"), followed by (ii) a phase with an approximately constant release rate with a stable daily release concentration of around $1 \mu \mathrm{g} \mathrm{ml}^{-1}$ (zero order kinetics). After 62 days, $15 \%$ of berberine was released. Chlorhexidine release concentration was too low to be detected with our

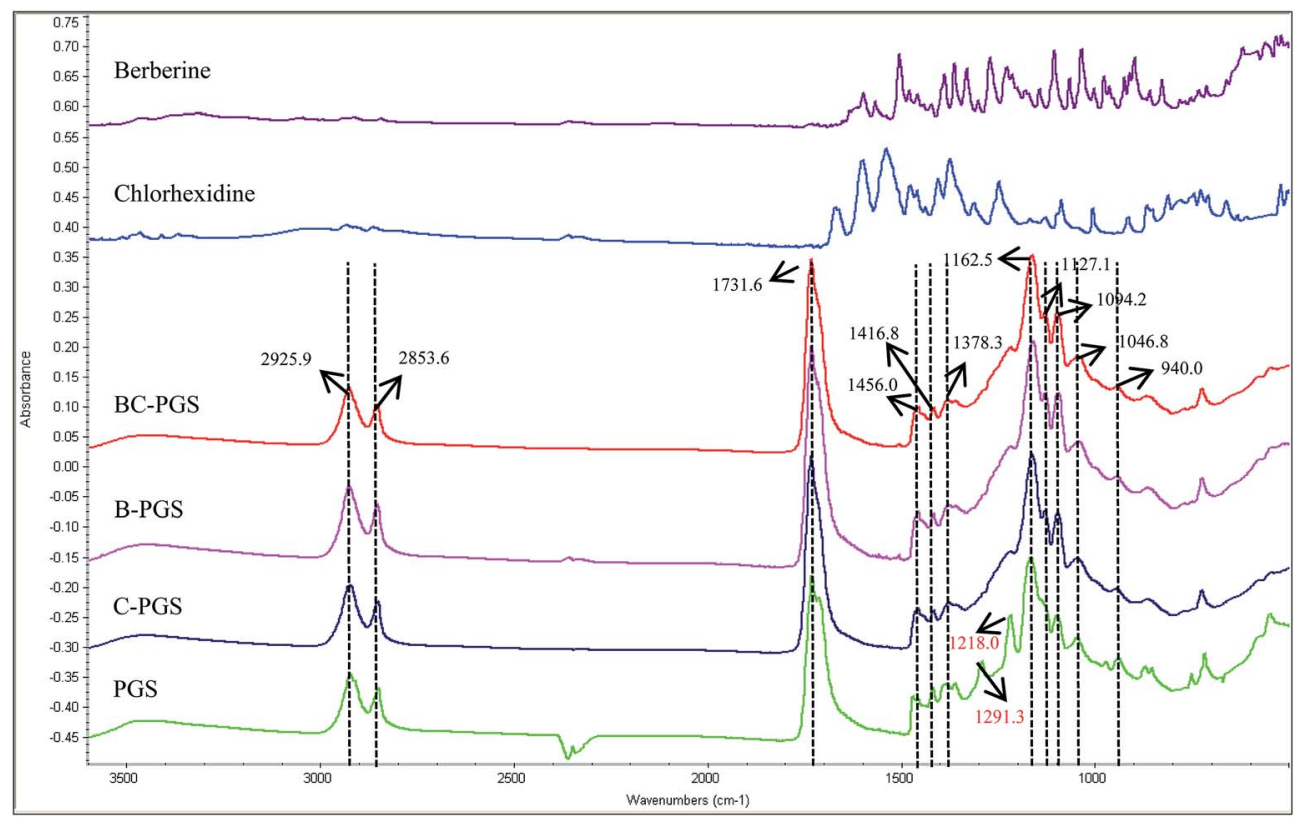

Fig. 1 FTIR spectra of berberine, chlorhexidine, BC-PGS, B-PGS, C-PGS, and PGS. 


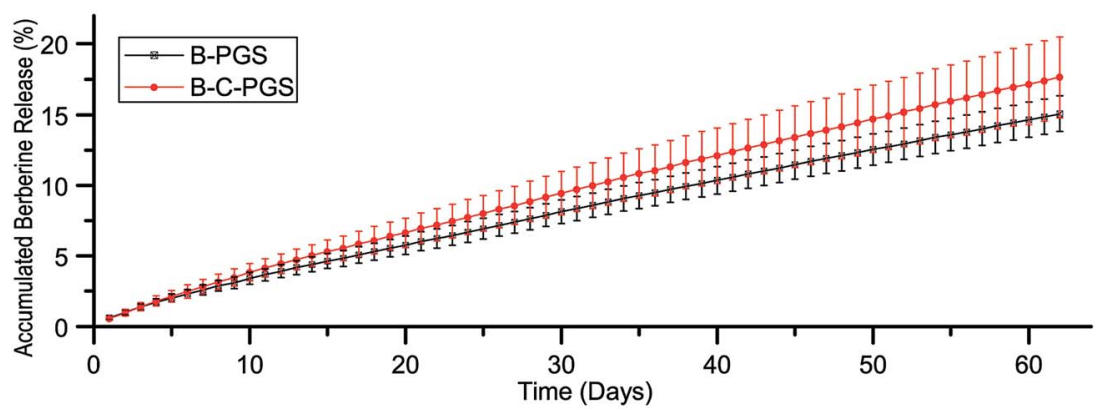

Fig. 2 Accumulated release of berberine in PBS.

method $\left(<5 \mu \mathrm{g} \mathrm{ml}{ }^{-1}\right)$. Although we did not do any further treatment after drug loading, drugs performed a sustained release in drug-loaded PGS.

\subsection{Mechanical test}

One thing we want to figure out after drug loading is whether drug loading treatment or loaded $\operatorname{drug}(\mathrm{s})$ changed the properties of the original polymer. One important property of PGS is its elastic property. Thus we took an elongation test to test if there is any changes in PGS mechanical property after drug loading. As shown in Fig. 3, PGS (drug loading treated), B-PGS, C-PGS and BC-PGS showed no significant difference with untreated (untreated PGS) in Young's modulus (Fig. 3A) and strain at break (Fig. 3B). This result indicated our swelling drug loading method and loaded drug(s) did not change the mechanical property of original PGS. The cross-linking density was $20.31 \mathrm{~mol} \mathrm{~m}^{-3}$.

\subsection{Surface wettability test}

Material surface wettability is an important property for tissue engineering. ${ }^{52}$ We tested the surface wettability of PGS, B-PGS, C-PGS and BC-PGS using water contact angle test as shown in Fig. 4. The contact angle of PGS is $53.5^{\circ}$, which is suitable for cell attachment. Hydrophilic drug berberine decreased water contact angle to $46^{\circ}$. This indicated an improved surface wettability and can have some beneficial in tissue engineering. On the other side, hydrophobic drug chlorhexidine increase water contact angle to $83.5^{\circ}$, making the surface of C-PGS more hydrophobic. Interestingly, when hydrophilic drug berberine and hydrophobic drug chlorhexidine loaded together, BC-PGS performed a similar water contact angle $\left(47.7^{\circ}\right)$ as B-PGS.

\subsection{Antimicrobial test}

Fig. 5 shows the result of the antimicrobial test for PGS, B-PGS, C-PGS and BC-PGS. Chlorhexidine loaded groups (C-PGS and BC-PGS) showed a good antimicrobial effect against typical Gram-positive bacteria $S$. aureus, typical Gram-negative bacteria E. coli and typical antibiotic-resistant bacteria MRSA.

C-PGS killed $99.86 \%$ S. aureus, $94.36 \%$ E. coli, and 99.96\% MRSA while BC-PGS killed $99.88 \%$ S. aureus, $96.13 \%$ E. coli and $100 \%$ MRSA after 4 hours of incubation. B-PGS does not show any significant antimicrobial effect in general antimicrobial test. However, in most test groups, BC-PGS showed higher antimicrobial effect than C-PGS.

Anaerobic bacteria $P$. gingivalis and $A$. actinomycetemcomitans are the most important pathogen in the generation of periodontal disease, and thus they are used in our test. ${ }^{7-10}$ The results showed that both chlorhexidine and berberine has antimicrobial activity against $P$. gingivalis and A. actinomycetemcomitans. A clear inhibition zone was observed around B-PGS and BC-PGS samples on the bacterial agar plate as shown in Fig. 6. A smaller inhibition zone can also be found around CPGS. It may be due to the fact that hydrophilic drug berberine spreads out faster than hydrophobic drug chlorhexidine dichloride.

\subsection{Cell compatibility test}

Human gingival fibroblast (HGF-1) was used because it is the most abundant cell in periodontal soft tissue. Fig. 7 shows the fluorescence images of HGF-1 cell grown on sample surfaces after 3 days. Compare to PGS group (Fig. 7A), more cells were found on the surface of B-PGS (Fig. 7B) and BC-PGS (Fig. 7D), while fewer cells were found on the surface of C-PGS (Fig. 7C). This shows berberine loaded PGS groups has improved cell compatibility with HGF-1.

\section{Discussion}

\subsection{Using PGS as a drug loading carrier}

PGS has been widely used in many tissue engineering applications since 2002 because of its good biocompatibility, biodegradable and elastic property. PGS has a 3-D cross-linked structure composited by glycerol and sebacate acid. Sebacic acid is a dicarboxylic acid with 8 hydrophobic methylene groups $\left(-\mathrm{CH}_{2}-\right)$ between two carboxylic groups, and glycerol is a highly hydrophilic polyol with three hydroxyl groups. In PGS, $\mathrm{C}_{8}$ methylene group from sebacate acid formed a hydrophobic core and oxygen containing functional groups like ester bond, carboxyl group, and free hydroxyl group formed hydrophilic part around the $\mathrm{C}_{8}$ hydrophobic core. In this way, hydrophobic drugs and hydrophilic drugs can both attach to PGS at different hydrophobicity site. Moreover, those oxygen-containing functional groups provided the possibility to generate hydrogen bond between the polymer and loaded drug, which can lead to more strong attachment. The 3-D cross-linked structure also 

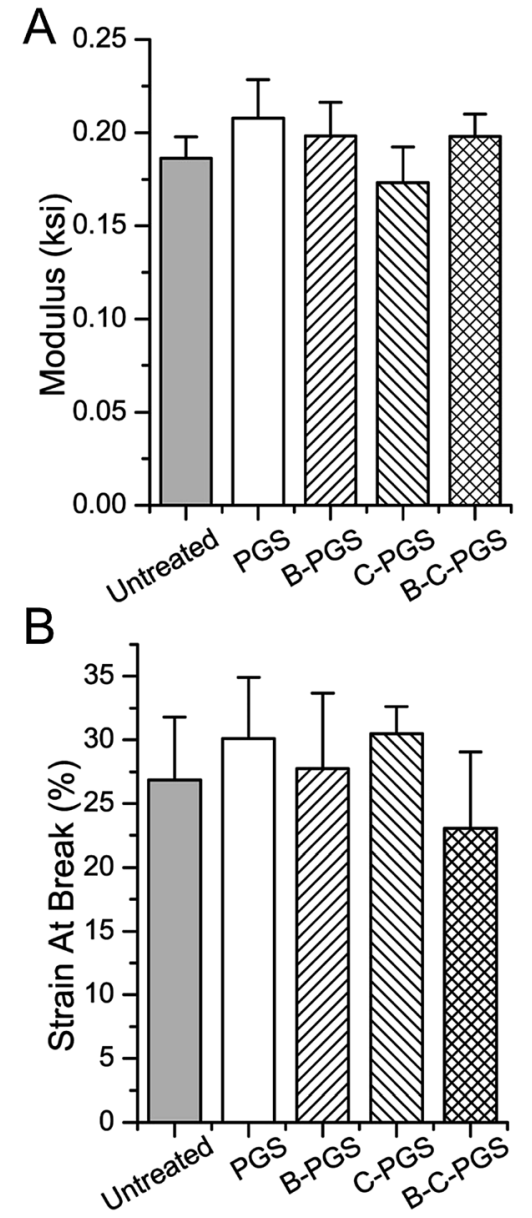

Fig. 3 Young's modulus (A) and strain at break (B) of untreated PGS (untreated), drug loading treated PGS (PGS), berberine loaded $(0.699 \%)$ PGS (B-PGS), chlorhexidine loaded (0.042\%) PGS (C-PGS) and berberine-chlorhexidine loaded PGS (BC-PGS).

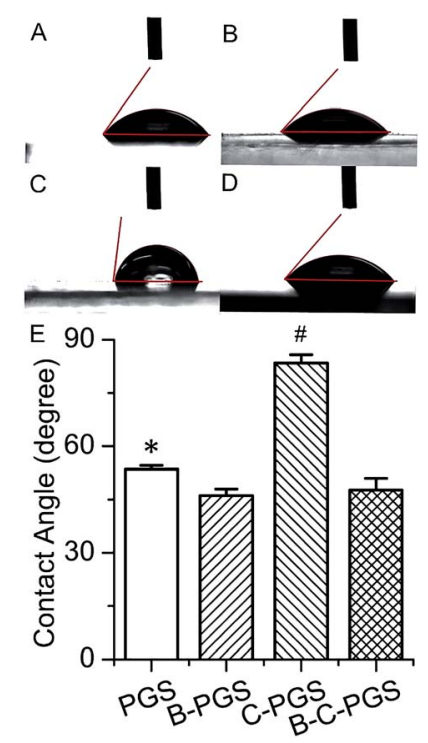

Fig. 4 Images ((A): PGS; (B): B-PGS; (C): C-PGS; (D): BC-PGS) and results of water contact angle test. (*. \# indicate the marked group has a significant difference to all other groups. $p<0.05$.) formed a network to easily trap drug molecules inside the polymer. All the points mentioned above made PGS an ideal drug carrier for both hydrophobic and hydrophilic drugs.

\subsection{Elastomer swelling and drug loading}

Sun et al. used PGS as a drug carrier to load 5-FU and curcumin. ${ }^{38,39}$ They loaded drug into PGS pre-polymer before the second-step synthesis. However, there are some limitations with their method: (1) loaded drugs will be treated with high temperature for a long period of time (24-72 hours) during the second-step synthesis. And this made their method not suitable for the heat-sensitive drug, which includes most antibiotics. (2) Organic solvents were applied to load drugs into pre-polymer. If any carboxyl, hydroxyl or amino groups from drug, organic solvent was left in the pre-polymer, those functional groups might react with PGS pre-polymer chain during the second step synthesis, and this will change the chemical structure of PGS.

Swelling is a general property for the elastomer. The swelling behavior has a tremendous influence on the diffusivity, making the polymer chains more flexible and resulting in an increased permeability. ${ }^{34}$ A greater swelling of the polymer allows relatively larger or more molecules to diffuse through its matrix. Thus the swelling can be used for drug loading application. According to our study, PGS is able to swell $55.22 \%(\mathrm{w} / \mathrm{w})$ in ethanol and $113.98 \%(\mathrm{w} / \mathrm{w})$ in acetone. It has even higher swell rate in other organic solvents like TFE. But it made PGS more fragile and easier to break. Swelling drug load is not widely used in polymer-based biomaterial, but it is a widely used method in hydrogel drug loading researches. ${ }^{53}$

The swelling drug loading has the following two mechanisms: (1) swelling and trapping, and (2) absorption and binding. In swelling and trapping mechanism, drugs load into the polymer matrix by diffusion and the rate of drug release dependent on the diffusion coefficient of the small molecule or macromolecule through the polymer network. So the drugs will be rapidly absorbed and rapidly eliminated. In absorption and binding mechanism, polymer interacts with the drug molecular, thus may increase the drug loading and slow down the drug release, which will proceed at a relatively constant rate. ${ }^{54,55}$ The estimated drug loading showed in Table 2 is calculated based on the swelling-trapping mechanism. Compare to the calculated value, the experimental results showed a much higher loading efficiency which indicated that absorption and binding mechanism should also be taken into account and PGS is able to absorb both hydrophilic berberine and hydrophobic chlorhexidine. The daily release concentration of both drugs showed a near-zero-order release pattern, which maintained in a constant rate for as long as 60 days. This proved our assumption that PGS has the ability to absorb and controlled release both hydrophilic and hydrophobic drugs. Drugs performed a sustained release in drug loaded PGS without any further treatment, and PGS itself is a good drug carrier.

\subsection{Behavior change before/after drug loading}

As a drug loading system, one thing need to be concerned is whether drug loading method or loaded drugs changed the 

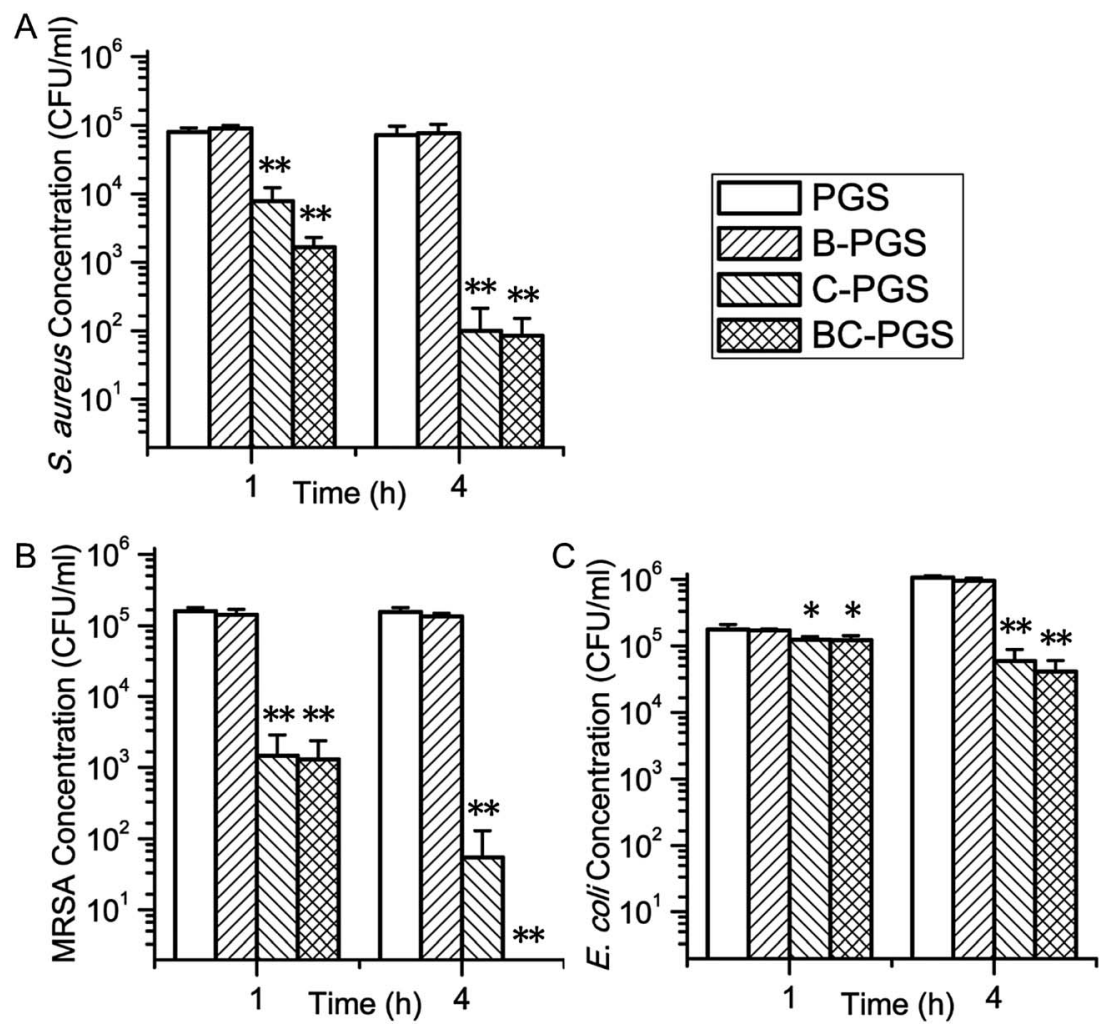

Fig. 5 General antimicrobial test of S. aureus (A), MRSA (B) and E. coli (C). (Star sign indicates the marked group has a significant difference to PGS group of each time point. $* p<0.05, * * p<0.01$.)

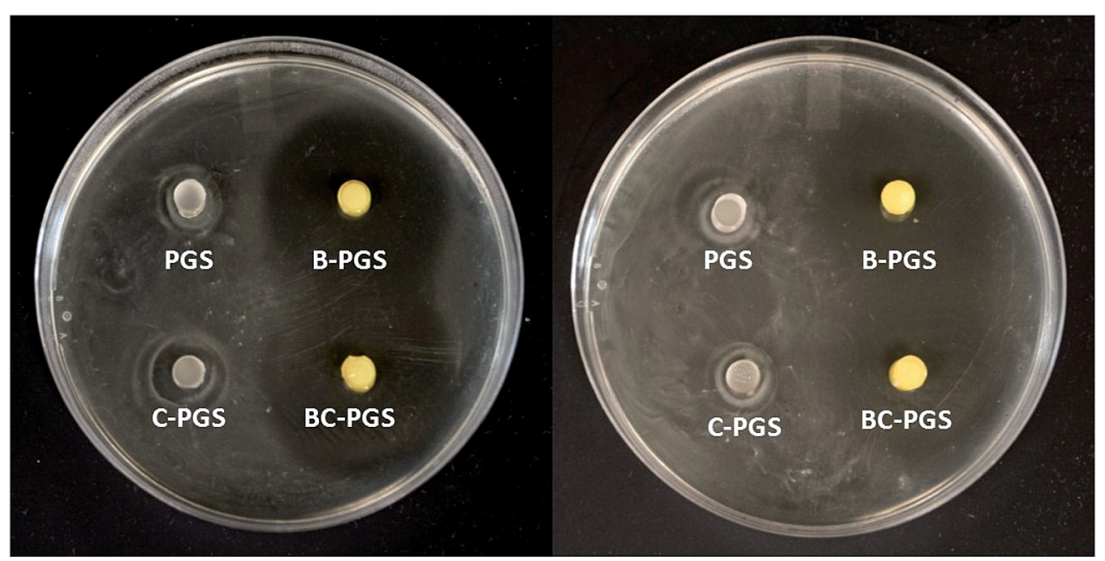

P. gingivalis

A. actinomycetemcomitans

Fig. 6 Inhibition zone of PGS, B-PGS, C-PGS and BC-PGS in P. gingivalis and A. actinomycetemcomitans plates.

original property of carrier. PGS is famous for its elastic property and good biological property in tissue engineering. According to our test, the mechanical property, in terms of Young's modulus and strain at break was not changed. Surface wettability is modified by loaded drug(s), while hydrophilic drug berberine made PGS more hydrophilic and hydrophobic drug chlorhexidine made PGS more hydrophobic. When berberine and chlorhexidine was loaded together, BC-PGS showed the same surface wettability as B-PGS. Two possible reasons can explain this: (1) hydrophilic drug berberine is more powerful in change surface wettability of PGS; and (2) the berberine content is much higher than chlorhexidine in BC-PGS.

\subsection{Drug release profile}

The initial drug release phase is primarily controlled by diffusion and the limited water solubility of the drug. However, the surface or near surface located drug was still diffused out very fast. ${ }^{56}$ In the second release phase, PGS polymer degradation 

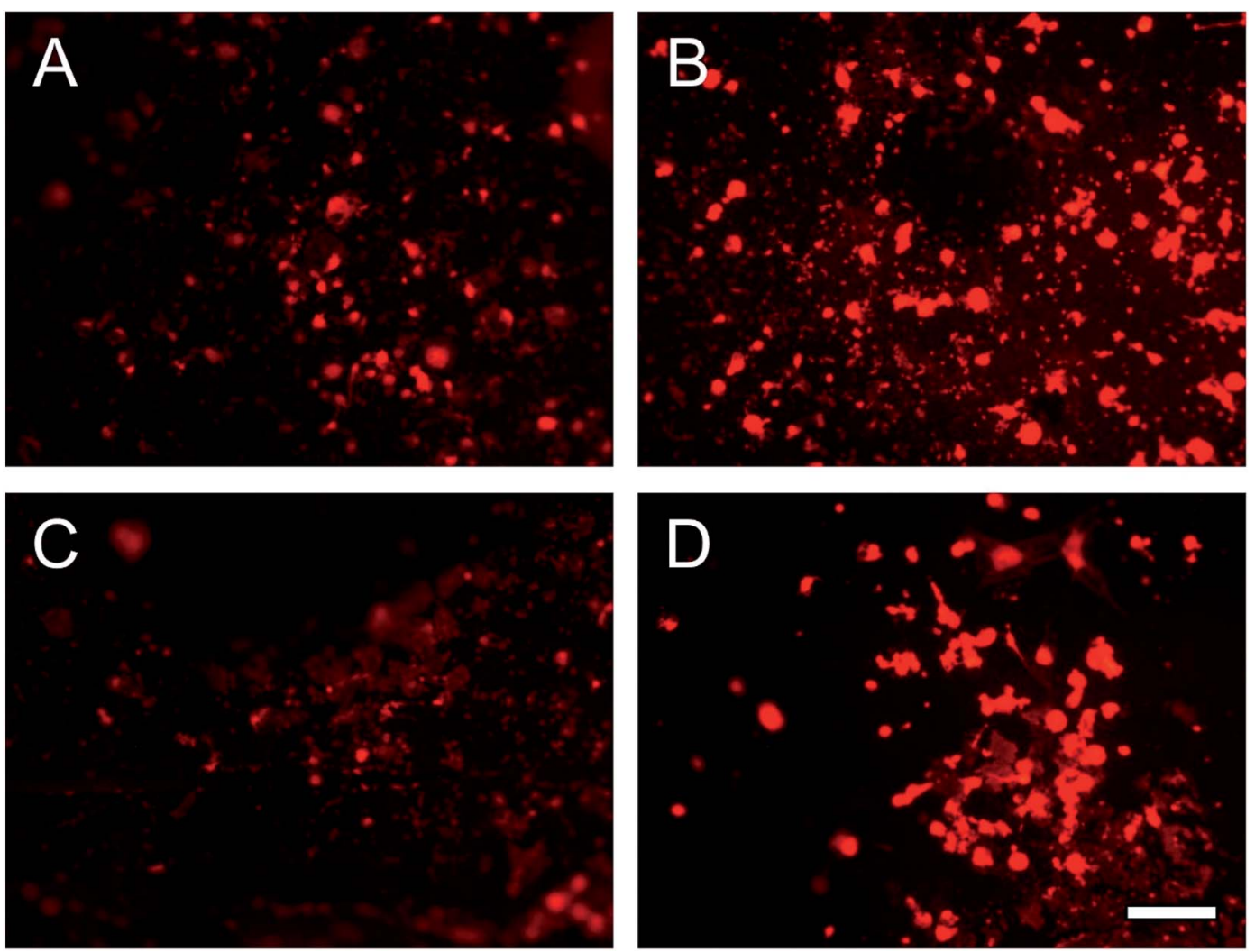

Fig. 7 Fluorescence images of HGF-1 cells on the surface of (A) PGS, (B) B-PGS, (C) C-PGS and (D) BC-PGS after three days of seeding. Scale bar indicates $200 \mu \mathrm{m}$.

becomes important. The increase in the length of the diffusion pathways with time should lead to a decrease in the release rate. ${ }^{57}$ However, this effect is compensated by the degradation of PGS polymer. Therefore, an increase in drug mobility was achieved. Further, PGS shows a linear in vivo and in vitro degradation profile and surface erosion pattern, resulting in significantly shortened diffusion pathways and an approximate of zero order release profile. ${ }^{31}$

\subsection{As periodontal disease treatment}

As shown in the antimicrobial test, C-PGS and BC-PGS performed excellent effect against typical Gram positive bacteria, Gram negative bacteria, and MRSA. Although C-PGS performed reduced cell compatibility, BC-PGS showed good cell compatibility with HGF-1 cell. All above made BC-PGS a good antimicrobial implant to be used in periodontal disease treatment.

According to the result of anti-periodontal bacteria test, a big inhibition zone was found around B-PGS and BC-PGS. This indicated berberine has good antimicrobial property against periodontal pathogens $P$. gingivalis and A. actinomycetemcomitans. Chlorhexidine is generally recognized as "gold standard" in the treatment of periodontal disease for its broad antimicrobial spectrum and high antimicrobial efficiency. Its toxicity and poor water solubility limited its use in some area. In BC-PGS, berberine would be likely to release to the surrounding environment because of its higher water solubility. Released berberine is able to generate a relatively sterile environment and to slow the periodontal tissue degradation process. It can also perform anti-inflammatory and tissue regeneration effect in large scale. ${ }^{58-60}$ Since PGS undergoes an enzymatic degradation in vivo, many enzymes from bacteria may cause or accelerate PGS degradation. As drugs were loaded in the PGS matrix, with PGS degradation, drugs may no longer bind to the polymer and therefore will be released to the surrounding environment. Where bacteria concentration is higher, PGS will degrade faster and release more chlorhexidine, which can effectively reduce local bacterial concentration. The poor solubility of chlorhexidine prevents its spreading and reduced its toxicity.

\section{Conclusion}

In our work, we successfully loaded drug(s) into PGS with swelling drug loading method. Drug loaded PGS kept its original mechanical property while the surface wettability, cell compatibility we have been modified. Among those drug loaded PGS, BC-PGS showed good antimicrobial property against Gram positive, Gram negative bacteria, MRSA, and periodontal pathogens. The cell compatibility of PGS remains good. All above indicated BC-PGS a good candidate to be used as an implant for the treatment of periodontal disease. 


\section{Acknowledgements}

This research is supported by South Dakota Board of Reagent and University of South Dakota Research \& Creative Activity Grants.

\section{References}

1 P. E. Petersen and H. Ogawa, J. Periodontol., 2005, 76, 21872193.

2 J. Highfield, Aust. Dent. J., 2009, 54(Suppl 1), S11-S26.

3 National Institutes of Health, Periodontal (Gum) Disease: Causes, Symptoms, and Treatments, NIH, NIH Publication No. 12-1142, 2012.

4 G. C. Armitage, Periodontology 2000, 2004, 34, 9-21.

5 M. Bansal, S. Rastogi and N. S. Vineeth, J. Med. Life, 2013, 6, 126-130.

6 S. S. Socransky and A. D. Haffajee, Periodontology 2000, 2005, 38, 135-187.

7 J.-H. Moon, C. Kim, H.-S. Lee, S.-W. Kim and J.-Y. Lee, J. Med. Microbiol., 2013, 62, 1307-1316.

8 M. C. Sánchez, M. J. Marín, E. Figuero, A. Llama-Palacios, D. Herrera and M. Sanz, J. Periodontal Res., 2012, 22957816.

9 L. Bonifait, A. Marquis, S. Genovese, F. Epifano and D. Grenier, Fitoterapia, 2012, 83, 996-999.

10 G. Prashant, R. B. Patil, T. Nagaraj and V. B. Patel, J. Contemp. Dent. Pract., 2012, 13, 749-752.

11 M. Sanz, J. Serrano, M. Iniesta, I. Santa Cruz and D. Herrera, Monogr. Oral Sci., 2013, 23, 27-44.

12 H. L. Wang, H. Greenwell, J. Fiorellini, W. Giannobile, S. Offenbacher, L. Salkin, C. Townsend, P. Sheridan, R. J. Genco, S. Research and C. Therapy, J. Periodontol., 2005, 76, 1601-1622.

13 A. Prakasam, S. S. Elavarasu and R. K. Natarajan, J. Pharm. BioAllied Sci., 2012, 4, S252-S255.

14 L. R. Johnson and N. H. Stoller, Comp. Cont. Educ. Dent., 1999, 20, 19-25.

15 J. Lessem and A. Hanlon, J. Int. Acad. Periodontol., 2004, 6, 150-153.

16 A. Stabholz, L. Shapira, D. Mahler, Y. Gellman, T. Ramon, E. Dolev, M. Schwartz, L. Berger, H. M. Proskin, R. D. Finkelman, M. Flashner, B. Kolatch and A. Soskolne, Comp. Cont. Educ. Dent., 2000, 21, 325-328.

17 C. G. Jones, Periodontology 2000, 1997, 15, 55-62.

18 J. Tang, Y. Feng, S. Tsao, N. Wang, R. Curtain and Y. Wang, J. Ethnopharmacol., 2009, 126, 5-17.

19 X. Wang, X. Yao, Z. Zhu, T. Tang, K. Dai, I. Sadovskaya, S. Flahaut and S. Jabbouri, Int. J. Antimicrob. Agents, 2009, 34, 60-66.

20 Q. Zou, Y. Li, L. Zhang, Y. Zuo, J. Li and J. Li, J. Biomed. Mater. Res., Part A, 2009, 89, 1108-1117.

21 X. Wang, S. Qiu, X. Yao, T. Tang, K. Dai and Z. Zhu, J. Orthop. Res., 2009, 27, 1487-1492.

22 M. L. Freile, F. Giannini, G. Pucci, A. Sturniolo, L. Rodero, O. Pucci, V. Balzareti and R. D. Enriz, Fitoterapia, 2003, 74, 702-705.
23 H. H. Yu, K. J. Kim, J. D. Cha, H. K. Kim, Y. E. Lee, N. Y. Choi and Y. O. You, J. Med. Food, 2005, 8, 454-461.

24 G. Tegos, F. R. Stermitz, O. Lomovskaya and K. Lewis, Antimicrob. Agents Chemother., 2002, 46, 3133-3141.

25 Q. Xie, B. R. Johnson, C. S. Wenckus, M. I. Fayad and C. D. Wu, J. Endod., 2012, 38, 1114-1117.

26 M. M. Kheir, Y. Wang, L. Hua, J. Hu, L. Li, F. Lei and L. Du, Food Chem. Toxicol., 2010, 48, 1105-1110.

27 J. Bao, B. Yang, Y. Sun, Y. Zu and Y. Deng, J. Biomed. Nanotechnol., 2013, 9, 1173-1180.

28 H. P. Tu, M. M. Fu, P. J. Kuo, Y. T. Chin, C. Y. Chiang, C. L. Chung and E. Fu, Phytomedicine, 2013, 20, 1203-1210.

29 Y. Wang, G. A. Ameer, B. J. Sheppard and R. Langer, Nat. Biotechnol., 2002, 20, 602-606.

30 A. Patel, A. K. Gaharwar, G. Iviglia, H. Zhang, S. Mukundan, S. M. Mihaila, D. Demarchi and A. Khademhosseini, Biomaterials, 2013, 34, 3970-3983.

31 Y. Wang, Y. M. Kim and R. Langer, J. Biomed. Mater. Res., Part A, 2003, 66, 192-197.

32 I. Pomerantseva, N. Krebs, A. Hart, C. M. Neville, A. Y. Huang and C. A. Sundback, J. Biomed. Mater. Res., Part A, 2009, 91, 1038-1047.

33 S. L. Liang, X. Y. Yang, X. Y. Fang, W. D. Cook, G. A. Thouas and Q. Z. Chen, Biomaterials, 2011, 32, 8486-8496.

34 X. D. Li, A. T. L. Hong, N. Naskar and H. J. Chung, Biomacromolecules, 2015, 16, 1525-1533.

35 J. L. Ifkovits, J. J. Devlin, G. Eng, T. P. Martens, G. VunjakNovakovic and J. A. Burdick, ACS Appl. Mater. Interfaces, 2009, 1, 1878-1886.

36 Q. Liu, T. Tan, J. Weng and L. Zhang, Biomed. Mater., 2009, 4, 025015.

37 R. Rai, M. Tallawi, A. Grigore and A. R. Boccaccini, Prog. Polym. Sci., 2012, 37, 1051-1078.

38 Z. J. Sun, C. Chen, M. Z. Sun, C. H. Ai, X. L. Lu, Y. F. Zheng, B. F. Yang and D. L. Dong, Biomaterials, 2009, 30, 5209-5214.

39 Z. J. Sun, B. Sun, R. B. Tao, X. Xie, X. L. Lu and D. L. Dong, J. Biomed. Mater. Res., Part A, 2013, 101, 253-260.

40 US Department of Health and Human Services, US Food and Drug Administration, Guidance for Industry Q3C - Tables and List, 2012, http://www.fda.gov/Drugs/ GuidanceComplianceRegulatoryInformation/Guidances/ default.htm.

41 K. Grodowska and A. Parczewski, Acta Pol. Pharm., 2010, 67, 3-12.

42 S. J. Kim, S. J. Park and S. I. Kim, React. Funct. Polym., 2003, 55, 53-59.

43 H. T. Peng, L. Martineau and P. N. Shek, J. Mater. Sci.: Mater. Med., 2007, 18, 975-986.

44 P. a. Westbrook and R. n. French, Polym. Eng. Sci., 2007, 47, 1554-1568.

45 N. Kundu, A. Roy, D. Banik and N. Sarkar, J. Phys. Chem. B, 2016, 120, 1106-1120.

46 N. J. Medlicott, D. G. Ferry, I. G. Tucker, M. J. Rathbone, D. W. Holborow and D. S. Jones, J. Liq. Chromatogr., 1994, 17, 1605-1620.

47 M. J. Kim, M. Y. Hwang, J. Kim and D. J. Chung, BioMed Res. Int., 2014, 956952, DOI: 10.1155/2014/956952. 
48 ASTM, Test Method for Determining the Antimicrobial Activity of Immobilized Antimicrobial Agents Under Dynamic, 2013, ASTM E2149 - 10.

49 C. Dahlin, A. Johansson, M. Hoffman and A. Molenberg, Clin. Oral Implants Res., 2014, 25, 16-20.

50 Y. S. Kim, Y. Ko, S. B. Kye and S. M. Yang, Int. J. Oral Maxillofac. Implants, 2014, 29, 969-975.

51 I. H. Jaafar, C. E. LeBlon, M. T. Wei, D. Ou-Yang, J. P. Coulter and S. S. Jedlicka, Acta Biomater., 2011, 7, 1588-1598.

52 B. N. Lourenco, G. Marchioli, W. Song, R. L. Reis, C. A. van Blitterswijk, M. Karperien, A. van Apeldoorn and J. F. Mano, Biointerphases, 2012, 7, 46.

53 B. L. Foss, T. W. Maxwell and Y. Deng, J. Mech. Behav. Biomed. Mater., 2014, 29, 56-67.
54 L. Li, L. Wang, Y. Shao, R. Ni, T. Zhang and S. Mao, Int. J. Pharm., 2013, 450, 197-207.

55 F. Rossi, F. Castiglione, M. Ferro, P. Marchini, E. Mauri, M. Moioli, A. Mele and M. Masi, ChemPhysChem, 2015, 16, 2818-2825.

56 T. W. Xu and B. L. He, Int. J. Pharm., 1998, 170, 139-149.

57 N. Faisant, J. Akiki, F. Siepmann, J. P. Benoit and J. Siepmann, Int. J. Pharm., 2006, 314, 189-197.

58 J. M. Hur and D. Kim, Toxicol. Res., 2010, 26, 109-115.

59 C. L. Kuo, C. W. Chi and T. Y. Liu, Cancer Lett., 2004, 203, 127-137.

60 B. Szotowski, S. Antoniak, P. Goldin-Lang, Q. V. Tran, K. Pels, P. Rosenthal, V. Y. Bogdanov, H. H. Borchert, H. P. Schultheiss and U. Rauch, Cardiovasc. Res., 2007, 73, 806-812. 